\title{
DENSIDAD POBLACIONAL Y ESTRUCTURA DE GRUPO DE Alouatta seniculus (PRIMATES: ATELIDAE) EN COLOSÓ, SUCRE, COLOMBIA
}

\section{POPULATIONAL DENSITY AND GROUP STRUCTURE OF Alouatta seniculus (PRIMATES: ATELIDAE) IN COLOSÓ, SUCRE, COLOMBIA}

\author{
David F. Ochoa¹, Edwin Martínez¹, Jaime De La Ossa 2
}

\begin{abstract}
${ }^{1}$ Biólogos Universidad de Sucre. ${ }^{2}$ Facultad de Ciencias Agropecuarias, Grupo de Investigación en Biodiversidad Tropical, Universidad de Sucre: Carrera 28 № 5-267, Puerta Roja, Sede Principal, Sincelejo, Sucre, Colombia. jaimedelaossa@yahoo.com.
\end{abstract}

Rev. U.D.C.A Act. E Div. Cient. 14(2): 101 - 108, 2011

\section{RESUMEN}

En este estudio, se determinó la densidad poblacional y la estructura de grupo para una población de Alouatta seniculus (L. 1766) (Primates: Atelidae), asentada en una zona fragmentada de los arroyos Colosó y Sereno, del Municipio de Colosó, en el Departamento de Sucre, Colombia. La zona de estudio, se ubicó en los Montes de María, formación de bosque seco tropical, que conforma un relictus en el cinturón árido Pericaribeño. Se aplicó el método seguimiento continuo, con 84 salidas de campo durante un año, entre 2009 y 2010. Se registraron 22 grupos en total, con una densidad de 52,4 Ind./ $/ \mathrm{km}^{2}$ y de 8,7 Grupos $/ \mathrm{km}^{2}$. La proporción entre machos y hembras fue de 1:1. Las variables poblacionales analizadas concuerdan con los rangos establecidos para la especie, pero difieren en cuanto a densidad y estructura de grupo con trabajos previos, llevados a cabo en la zona. Se evidencia un modelo socioecológico, que favorece el incremento de la densidad, mediante la conformación de múltiples grupos de menor tamaño.

Palabras clave: Alouatta seniculus, densidad, estructura grupal, Sucre, Colombia.

\section{SUMMARY}

The populational density and group structure for a Alouatta seniculus (Primates: Atelidae) population seated in a fragmented area of the Colosó and Sereno streams in the
Municipality of Colosó, Department of Sucre, Colombia, was determined. The study area was located in Montes de Maria, dry tropical dry formation that structure a relictus in the arid Caribbean region. The method of continuous pursuits was applied during 84 field days during one year, between 2009 and 2010. Twenty two groups in total, with a density of 52.4 Ind. $/ \mathrm{km}^{2}$, and of 8.7 Groups $/ \mathrm{km}^{2}$ were registered. The proportion between males and females was 1:1. The populational variables analyzed agree with the established ranges for the species, but they differ for density and group structure according to previous studies carried out in the area. A socioecological model was evidenced, favoring the density increase through the conformation of multiple smaller sized groups.

Key words: Alouatta seniculus, density, group structure, Sucre, Colombia.

\section{INTRODUCCIÓN}

Colombia es el quinto país con mayor diversidad de primates del nuevo mundo, presentando 12 de los 16 géneros existentes, distribuidos desde el nivel del mar hasta los 3.200msnm (Defler, 2003). No obstante, la transformación de hábitat y el acrecentamiento de la frontera agrícola muestran su impacto. Es así como en la región Caribe, la antigua y la amplia cobertura de bosque seco tropical hoy corresponde, en su mayoría, a etapas sucesionales secundarias y sabanas antrópicas, con pequeños parches boscosos (Andrade $\mathcal{E}$ Mejía, 1998), afectando las poblaciones silvestres naturales 
y, entre ellas, a los primates, como sucede en todo el Neotrópico (Neville, 1976; Thorington \& Heltne, 1976; Defler, 1981; Stoner, 1994).

Con los procesos de fragmentación, las especies típicas del bosque quedan aisladas y su supervivencia obedece a la dinámica poblacional, a nivel del fragmento. Así, a escala local, el efecto del tamaño del fragmento, el efecto de borde y el efecto del aislamiento son los principales mecanismos de extinción poblacional; al quedar confinada a un fragmento, la probabilidad de una especie de persistir dependerá del tamaño y de la dinámica de la población; mientras más pequeña sea la población, mayor será su probabilidad de extinguirse (Kattan E Álvarez-López, 1996). En Sur América y en Colombia Alouatta seniculus es considerado en la categoría de bajo riesgo-preocupación menor (LR), especie que es capaz de persistir en bosques degradados y fragmentos (Defler, 2003).

De forma específica, los factores que afectan las poblaciones de $A$. seniculus, son: alteración del hábitat, con el consecuente aislamiento en parches de bosque y con limitaciones de alimentos; crecimiento reducido y baja resistencia a enfermedades, lo que puede, eventualmente, llegar a declinar poblacionalmente (Hershkovitz, 1949; Heltne et al. 1976); sin embargo, se le reconoce por su gran habilidad para sobrevivir en ambientes degradados, es altamente adaptable y capaz de utilizar pequeños parches de bosque de crecimiento secundario (Hernández-Camacho \& Cooper, 1976). Varias especies de primates están predispuestas a un incremento en su riesgo de extinción cuando sus hábitats se han modificado (Chapman E Peres, 2001); muchas especies de Primates presentan una gran plasticidad ecológica, la fragmentación de su hábitat los hace vulnerables al parasitismo y enfermedades, depredación y depresión genética (Bicca-Marques, 2003).

Alouatta seniculus es el primate de más amplia distribución en Colombia. Originalmente, se le reporta en todo el país, a excepción de la costa Pacífica, el desierto de la península de la Guajira y el suroccidente de los Andes (Nariño). Esta especie ocupaba todos los rangos altitudinales de la cordillera hasta los 3.200 msnm (regiones montañosas ubicadas sobre el nivel del bosque nublado) y la Sierra Nevada de Santa Marta (Hernández-Camacho \& Cooper, 1976). En los Montes de María (Sucre), no se ha mantenido un continuo estudio o monitoreo de sus poblaciones, a pesar de contar con la Estación Primatológica de Colosó; los últimos estudios reportados corresponden, principalmente, a la década comprendida entre 1986 y 1996 (Cuervo et al. 1986; Fajardo E De La Ossa, 1994; De La Ossa \& Fajardo, 1996).

En este trabajo, se determinan aspectos poblacionales de los grupos de A. seniculus presentes en los arroyos Colosó y Sereno, que hacen parte de la Reserva Forestal Protectora Serranía de Coraza y Montes de María, abordando un estudio de densidad y de composición de grupo, dentro de una matriz boscosa, que acusa una alta intervención y que se estructura, mayoritariamente, en parches de crecimiento secundario.

\section{MATERIALES Y MÉTODOS}

Área de estudio: Departamento de Sucre, municipio

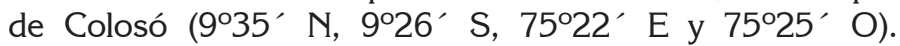
Puntualmente, en los arroyos Colosó y Sereno, que discurren a lo largo de la zona limítrofe con la ladera Sur de la serranía de San Jacinto ( $9^{\circ} 30^{\prime} \mathrm{N}$ y $75^{\circ} 22^{\prime} \mathrm{O}$ ). Ecológicamente, esta zona constituye un relicto de transición de bosque seco Premontano a bosque seco Tropical, con una altura máxima de $300 \mathrm{msnm}$, una temperatura media anual de $27^{\circ} \mathrm{C}$ y una pluviometría que alcanza los $1.200 \mathrm{~mm}$ anuales, lo que determina dos épocas climáticas al año: lluviosa, desde mediados de abril hasta finales de noviembre; seca, desde diciembre hasta comienzos de abril (IGAC, 1969; Cuervo et al. 1986).

Fase preliminar: Se realizó el reconocimiento del área donde se localizaban los grupos o manadas a estudiar, utilizando seguimiento continuo (Altmann, 1974), se geoposicionaron los dormideros y los lugares de alimentación y descanso (GPS Garmin 12X). Se trabajó con un esfuerzo total de 173 horas, correspondientes a 24 días de muestreo, ocho días continuos por cada fragmento, entre las 05:00 y 18:00 horas, con promedio de 7,2 horas/día.

La zona de estudio, se dividió en tres fragmentos de bosque, identificadas como A, B y C. Los dos primeros ubicados en el arroyo Colosó y el tercero conformado por ambos cuerpos de agua. Cada fragmento tuvo un área de: $A=0,39 \mathrm{~km}^{2}, B=$ $0,60 \mathrm{~km}^{2}$, y C $=1,55 \mathrm{~km}^{2}$ (Figura 1, Tabla 1).

Fase de campo: El estudio, se llevó a cabo entre mayo de 2009 y abril de 2010, con 84 días de campo. Se trabajó una semana por mes con seguimiento continuo (Altmann, 1974) durante la fase diurna (06:00 a 16:00 horas), con promedio de 7,45 horas de trabajo/día, para un total de 626 horas de esfuerzo muestreal, en un área total de 2,14 km² (214 ha).

Estimación de la densidad: Se efectuó mediante conteo directo de los individuos y grupos en cada fragmento, teniendo en cuenta el tamaño manejable del área total (Cuervo et al. 1986; Fajardo \& De La Ossa, 1994; De La Ossa E Fajardo, 1996). Las diferencias individuales, se establecieron teniendo en cuenta características morfológicas visibles, 


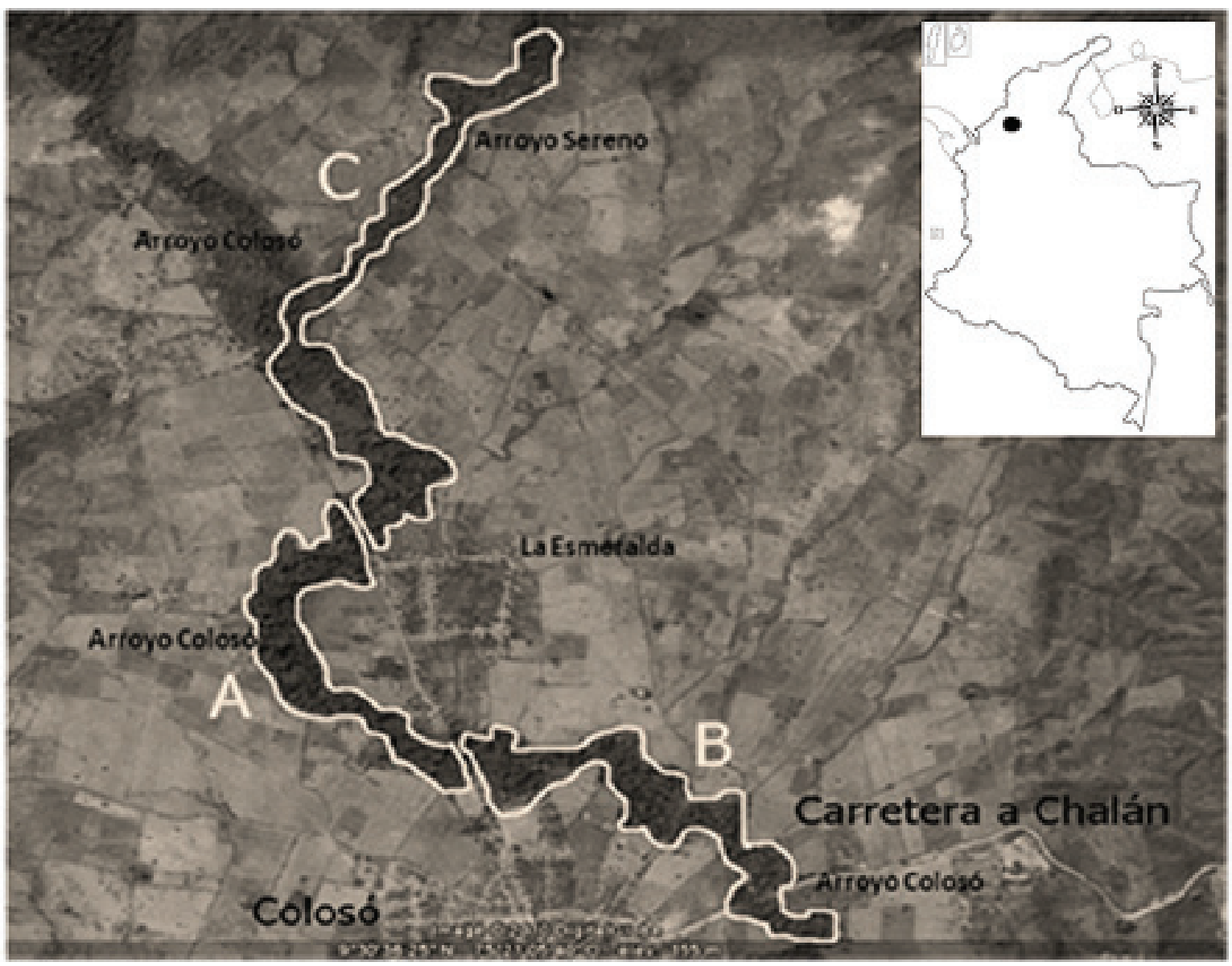

Figura 1. Imagen satelital de los fragmentos de bosques estudiados en Colosó, Sucre, Colombia (Google Earth 5.0 Free,

Tabla 1. Abundancia y densidad de A. seniculus en los tres fragmentos de los arroyos Colosó y Sereno.

\begin{tabular}{|c|c|c|c|c|c|c|}
\hline Fragmento & $\begin{array}{c}\text { No. de } \\
\text { individuos }\end{array}$ & $\begin{array}{c}\text { No. de } \\
\text { grupos }\end{array}$ & Área $\left(\mathrm{km}^{2}\right)$ & $\begin{array}{c}\text { Densidad } \\
\left(\text { Ind./km²) }^{2}\right.\end{array}$ & $\begin{array}{c}\text { Densidad } \\
(\text { Grupos/km²) }\end{array}$ & $\begin{array}{c}\text { Esfuerzo } \\
\text { muestreo (horas) }\end{array}$ \\
\hline A & 36 & 7 & 0,39 & 92,3 & 17,9 & 228 \\
\hline B & 70 & 10 & 0,60 & 116,7 & 16,7 & 265 \\
\hline C & 27 & 5 & 1,55 & 17,4 & 3,2 & 133 \\
\hline Total & 133 & 22 & 2,54 & 52,4 & 8,7 & 626 \\
\hline
\end{tabular}

mediante binoculares, tales como: patrones de coloración de la espalda, cola y extremidades, cicatrices, forma de las cejas y despigmentación o manchas (Escobedo-Morales $\varepsilon$ Mandujano, 2007); además, se tuvo en cuenta la localización espacial de las tropas (De La Ossa E Fajardo, 1996). La densidad de población fue calculada como densidad cruda o absoluta: el número de individuos dividido por el área total de la localidad (Soini, 1992).
Estructura y composición de los grupos: Durante el conteo de los grupos, se registró el número de individuos en cada tropa, teniendo en cuenta estado de madurez y el sexo, tomando como estadios: hembra y macho, para adultos y juvenil e infante, para inmaduros (Defler, 1981; Soini, 1992). Para la diferenciación de ambos sexos, se tuvo en cuenta el dimorfismo de los genitales externos y para determinar a las hembras, la presencia de crías o la lactancia; para diferenciar 
a los juveniles de los infantes, se observó la dependencia relativa materna (Defler, 1981; Cuervo et al. 1986; Soini, 1992).

Tratamiento de la información: Para analizar, si cada una de las variables evaluadas presentaba diferencias significativas entre los tres fragmentos, se hizo ANOVA y pruebas de Kruskal Wallis (Zar, 1996). Para el análisis e interpretación de los resultados obtenidos durante la investigación, se organizaron tablas y gráficas.

\section{RESULTADOS Y DISCUSIÓN}

En total, se registraron 22 grupos de $A$. seniculus. La densidad general, se calculó en $52,4 \mathrm{Ind} / \mathrm{km}^{2}$, con una participación de 8,7 grupos/ $\mathrm{km}^{2}$. Las variaciones numéricas entre fragmentos, se exponen en la tabla 1. Al comparar estadísticamente los resultados poblacionales entre áreas, se determinó que existe diferencia significativa $(p<0,05)$.
El tamaño promedio de las tropas fue de 6,0 \pm 2 y varió desde tres hasta once individuos (Tabla 2). La composición media por sexos y por edad fue de $30,1 \%$, machos adultos; $34,6 \%$, hembras adultas; 6,8\%, machos juveniles; $8,3 \%$, hembras juveniles y $16,5 \%$, infantes. Los grupos poseían de uno a tres machos adultos, una a cinco hembras adultas y de uno a tres individuos inmaduros (juveniles e infantes). En general, se halló igual proporción de hembras y de machos por grupo $(1: 1)(p<0,05)$. La distribución por estado de madurez y de sexo, no tuvo variación entre fragmentos, en su orden: machos: hembras: infantes, hallada fue: fragmento A: 4,7: 3,3: 2; fragmento B: 3,4: 4,6: 2; fragmento C: 4,4: 4,8: 0,8 .

La densidad hallada en este estudio, se halla dentro de los rangos reportados para la especie (Neville et al. 1976; Gaulin E Gaulin, 1982; Crockett \& Eisenberg, 1987; Stevenson, 1991; Soini, 1992; Morales-Jiménez, 2002; Defler, 2003). En general, pueden existir variaciones en los rangos de

Tabla 2. Tamaño y composición de los grupos de A. seniculus en los tres fragmentos de los arroyos Colosó y Sereno.

\begin{tabular}{|c|c|c|c|c|c|c|c|}
\hline \multirow{3}{*}{$\begin{array}{c}\text { Grupo } \\
\text { A1 }\end{array}$} & \multicolumn{2}{|c|}{ Adulto } & \multicolumn{2}{|c|}{ Juvenil } & \multirow{3}{*}{$\begin{array}{c}\text { Infante } \\
1\end{array}$} & \multirow{3}{*}{$\begin{array}{c}\begin{array}{c}\text { Total } \\
\text { Ind./grupo }\end{array} \\
6\end{array}$} & \multirow{3}{*}{$\begin{array}{c}\text { Total } \\
\text { Ind./fragmentc }\end{array}$} \\
\hline & o & q & $\hat{0}$ & q & & & \\
\hline & 1 & 1 & 2 & 1 & & & \\
\hline A2 & 2 & 2 & & 1 & 2 & 7 & \\
\hline A3 & 3 & & & & & 3 & \\
\hline A4 & 3 & 3 & & & 1 & 7 & \\
\hline A5 & 2 & 1 & & & 1 & 4 & \\
\hline A6 & 3 & & & & & 3 & \\
\hline A7 & 1 & 2 & & 1 & 2 & 6 & 36 \\
\hline B1 & 1 & 2 & & & 1 & 4 & \\
\hline B2 & 2 & 3 & 1 & 2 & 2 & 10 & \\
\hline B3 & 2 & 2 & 2 & & 1 & 7 & \\
\hline B4 & 2 & 5 & 1 & & 3 & 11 & \\
\hline B5 & 2 & 3 & & & & 5 & \\
\hline B6 & 2 & 3 & 1 & & 2 & 8 & \\
\hline B7 & 2 & 2 & & 2 & 2 & 8 & \\
\hline B8 & 3 & 2 & & & 1 & 6 & \\
\hline B9 & 1 & 2 & & 1 & 1 & 5 & \\
\hline B10 & 2 & 3 & & & 1 & 6 & 70 \\
\hline C1 & 3 & 2 & & 1 & 1 & 7 & \\
\hline $\mathrm{C} 2$ & 1 & 2 & & & & 3 & \\
\hline $\mathrm{C} 3$ & 1 & 2 & 1 & & 1 & 5 & \\
\hline $\mathrm{C} 4$ & 3 & 2 & & 2 & & 7 & \\
\hline C5 & 2 & 2 & 1 & & & 5 & 27 \\
\hline Total & 44 & 46 & 9 & 11 & 22 & 133 & \\
\hline Media Ind./grupo & 2,0 & 2,3 & 1,3 & 1,4 & 1,4 & 6,0 & \\
\hline
\end{tabular}


densidad, estas diferencias se atribuyen a factores de calidad de hábitat (Cuervo et al. 1986; Defler, 1981).

Los resultados para el mismo lugar y con la misma aplicación metodológica muestran, en un lapso de 15 años, un incremento de la densidad del $133,2 \%$, tomando en cuenta el último reporte llevado a cabo por Fajardo $\varepsilon$ De La Ossa (1994), en donde se estableció una densidad de 22,47 Ind./ $\mathrm{km}^{2}$.

Se sugiere que en áreas fragmentadas, las altas densidades de primates son producto del hacinamiento de la población al reducirse su área de ocupación original (Defler, 1981). Por otro lado, factores sociales, como la época de violencia vivida en la zona y que concuerda casi con el lapso de 15 años, entre los datos analizados, creó un ambiente de menor impacto antrópico sobre las poblaciones objeto de estudio; cabe resaltar que no se tienen evidencias históricas ni actuales de cacería o de utilización de esta especie en la zona (Cuervo et al. 1986; Fajardo E De La Ossa, 1994). No hubo un incremento significativo del área boscosa, pero sí una menor o casi nula circulación humana y se detuvo la extracción de maderas y la ampliación de áreas agrícolas. A. seniculus usualmente desaparece en áreas donde existen profundos disturbios en el hábitat, pero es considerado adaptable a la intromisión humana (Hernández-Camacho $\mathcal{E}$ Cooper, 1976).

Igualmente, aspectos como la tala de bosques restringe A. seniculus a los parches remanentes de bosque, lo cual, podría generar una importante concentración poblacional (Neville, 1976; Neville et al. 1976; Peres, 1990; Crockett, 1998; Laurance et al. 2002). Altas densidades de A. seniculus que habitan parches de bosques también se asocian a la restricción que impone la fragmentación y que impide la dispersión de los individuos jóvenes (Gómez-Posada et al. 2005; Valderrama \& Kattan, 2006); ahora, si los fragmentos mantienen su productividad, la capacidad de carga se estabiliza alcanzando su máxima expresión, mostrando la densidad una relación directa con las estructuras antrópicas existentes para el lugar (Estrada \& Coates-Estrada, 1988; Valderrama \& Kattan, 2006).

Por otro lado, es importante resaltar que este hallazgo, en el que la estrategia está dada por el incremento de grupos y no por el tamaño del grupo, permite aceptar la existencia de un modelo de dispersión in situ para A. seniculus, que brinda una mayor eficiencia en el aprovechamiento de los recursos disponibles y en el mantenimiento de la población.

Puesto que en Primates los grupos grandes invierten mayor tiempo en forrajeo, hacen recorridos mayores, las tasas de agresión intragrupal se incrementan y se aumenta el esfuerzo de crianza, en tal sentido, serían ecológicamente menos eficientes (Terborgh $\mathcal{E}$ Janson, 1986; Izawa, 1988; Janson, 1988; Janson \& van Schaik, 1988; Lee, 1994; Janson E Goldsmith, 1995; van Schaik, 1989), pudiéndose aceptar que, ante la limitación de hábitat para A. seniculus, sería más eficiente un modelo caracterizado por la presencia de múltiples grupos pequeños, que le permitan maximizar su eficacia.

La composición macho: hembra de los grupos estudiados es proporcionalmente menor a la reportada para esta especie tanto en hábitats similares como diferentes (<biblio $>$ ). Por lo general, se espera una ligera mayor proporción de hembras adultas que de machos adultos, (Defler, 1981; Gómez-Posada et al. 2009). Este trabajo no determinó una diferencia significativa entre la proporción de hembras y de machos, como existe en otros reportes (Izawa, 1988; Soini, 1992), pero se asemeja a lo establecido para la misma zona de estudio en cuanto al tamaño promedio del grupo y a la proporción entre sexos (Fajardo \& De La Ossa, 1994), datos que están de acuerdo con lo establecido por diversos autores para Perú y para Colombia (Freese, 1975; Klein E Klein, 1976; Neville et al. 1976).

Como lo plantean Valderrama \& Kattan (2006), la similitud de proporción entre sexos para esta especie puede ser explicada, porque el número de hembras adultas es el factor que, al parecer, limita el tamaño del grupo; las hembras adultas regulan su número expulsando a las hembras jóvenes; las tropas con muchas hembras adultas, atraen en mayor proporción machos solitarios que intentan ingresar al grupo y derrotar al macho dominante, generando cambios en la estructura y en la estabilidad social de la manada. Se podría explicar como una consecuencia del incremento del número de grupos que, comparativamente, se registró en este trabajo; esto evidenciaría la importancia de las interacciones sociales y las condiciones ecológicas (Winterhalder $E$ Smith, 1992); en este caso, las estrategias sociales se pueden relacionar con soluciones adaptativas a ciertas condiciones ecológicas específicas, como la limitación de hábitat (Chan, 1996) y que se hacen notorias en la organización social (Sterck et al. 1997).

Es evidente que se presentó un cambio histórico que favoreció la densidad poblacional para el área de estudio; sin embargo, el tamaño y la composición del grupo no manifiestan mudanzas significativas; se trata, entonces, de un incremento del número de grupos bajo la misma estructura poblacional. Durante estos 15 años, se han conformado nuevas manadas dentro de la misma área de estudio, lo que determina el incremento de la densidad poblacional. Es necesario continuar evaluando esta zona, en particular 
para determinar los umbrales de tolerancia de la especie con relación a los efectos de borde, aislamiento y tamaño de los fragmentos, y a los factores antrópicos asociados (Roncancio et al. 2010).

La información analizada no permite aseverar que la población se encuentre en riesgo de extinción local; se puede asegurar la existencia de una recuperación numérica importante, pero queda por definir la tolerancia de la capacidad de carga existente; es importante calcular su umbral máximo, conocer si ya llegó a su límite y qué podría suceder a futuro con esta área de fragmentos boscosos, que hacen parte de una Reserva Forestal Protectora de bosque seco tropical, remanente para el Caribe colombiano.

Conflicto de intereses: El manuscrito fue preparado y revisado con la participación de todos los autores, quienes declaramos que no existe ningún conflicto de intereses que ponga en riesgo la validez de los resultados presentados. Financiación: Este estudio fue financiado con aportes del Grupo de Investigación en Biodiversidad Tropical de la Universidad de Sucre, Colombia.

\section{BIBLIOGRAFÍA}

1. ALTMANN, J. 1974. Observational study of behavior sampling methods. Behaviour. 49(3-4):227-267.

2. ANDRADE, G.I.; MEJÍA, C. 1998. Cambios estacionales en la distribución de la avifauna terrestre en el Parque Nacional Natural Macuira, Guajira, Colombia. Trianea. 1:145-169.

3. BICCA-MARQUES, J.C. 2003. How do howler monkeys cope with habitat fragmentation? En: Primates in fragments, ecology and conservation. En: Marsh, L.K. ed. Kluwer Academy/Plenum Publishers. p.283-303.

4. CHAN, L.K.W. 1996. Phylogenetic interpretations of primate socioecology: With special reference to social and ecological diversity in Macaca. En: Martins, E.P. ed. Phylogenies and the Comparative Method in Animal Behavior. Oxford University Press (New York). p.324360.

5. CHAPMAN, C.; PERES, C. 2001. Primate conservation in the new millennium. The role of scientists. Evol. Anthropol. 10:16-33.

6. CROCKETT, C.; EISENBERG, J. 1987. Howlers: variations in group size and demography. En: Smuts, B.; Cheney, D.; Seyfarth, R.; Wrangham, R.; Struhsaker. T. eds.
Primate Societies. The University of Chicago Press (Chicago). p.54-68.

7. CROCKETT, C. 1998. Conservation biology of the Genus Alouatta. Int. J. Primatol. 19(3):549-578.

8. CUERVO, A.; BARBOSA, C.; DE LA OSSA, J. 1986. Aspectos ecológicos y etológicos de primates con énfasis en Alouatta seniculus (CEBIDAE) de la Región de Colosó, Serranía de San Jacinto (Sucre) Costa Norte Colombiana. Caldasia. XIV:68-70.

9. DE LA OSSA, J.; FAJARDO, P.A. 1996. Densidad del mono aullador Alouatta seniculus (Linnaeus, 1766) Mammalia: Primates. En el arroyo Colosó, Departamento de Sucre, Colombia. Acta Biol. Col. 3:43-52.

10. DEFLER, T. 1981. The density of Alouatta seniculus in the eastern llanos of Colombia. Primates. 22:564-569.

11. DEFLER, T. 2003. Primates de Colombia. Serie de guías tropicales de Colombia 4. Conservación Internacional. Bogotá, Colombia. 543p.

12. ESCOBEDO-MORALES, L.; MANDUJANO, S. 2007. Viabilidad metapoblacional del mono aullador (Alouatta palliata mexicana) en un paisaje altamente fragmentado de los Tuxtlas, México. En: Harvey, C.A.; Sáenz, J.C. eds. Evaluación y Conservación de biodiversidad en paisajes fragmentados Mesoamericanos. INBIO, CATIE, UNA (Costa Rica). p.421-450.

13. ESTRADA, A.; COATES-ESTRADA, R. 1988. Tropical rain forest conversion and perspectives in the conservation of wild primates (Alouatta and Ateles) in México. Am. J. Primatol. 14:315-327.

14. FAJARDO, P.A.; DELAOSSA, J. 1994. Censo Introductorio de primates en la reserva forestal protectora Montes de María y Serranía de Coraza, Sucre - Colombia. Trianea. 5:289-303.

15. FREESE, C.H. 1975. A census of non human primates in Perú. En: Project AMOR-0719. ed. Censusing Studies in Perú and Colombia. Report to National Academy of Sciences on Pan American Health Organization (Washington). p.17-41.

16. GAULIN, S.; GAULIN, C. 1982. Behavioral ecology of Alouatta seniculus in Andean Cloud Forest. Int. J. Primatol. 3(1):1-29. 
17. GÓMEZ-POSADA, C.; RONCANCIO, N.; HINCAPIÉ, P.; BETANCOURT, A. 2005. Densidad y composición de grupos en tres poblaciones de mono aullador rojo (Alouatta seniculus) en Valle y Cauca, Colombia. Bol. Cient. Mus. Hist. Nat. 14(1):79-91.

18. GÓMEZ-POSADA, C.; ÁlVAREZ, Z.; GIRALDOCHAVARRIAGA, P. 2009. Densidad y estatus poblacional de monos aulladores rojos en un guadual, fragmento aislado, La Tebaida, Quindío, Colombia. Universitas Scientiarum. 14(1):8-15.

19. HELTNE, D.; TURNER, D.; SCOTT, N. 1976. Comparison of census on Alouatta palliata from Costa Rica and Panamá. En: Thorington, R.W.; Heeltne, P.G. eds. Neotropical Primates: Field Studies and Conservation. National Academy of Sciences (Washington). p.10-19.

20. HERNÁNDEZ-CAMACHO, J.; COOPER, R.W. 1976. The nonhuman Primates of Colombia. En: Thorington, R.W.; Heeltne, P.G. eds. Neotropical Primates: Field Studies and Conservation. Nal Acad. Sci. (Washington). p.35-69.

21. HERSHKOVITZ, P. 1949. Mammals of northern Colombia. Preliminary report No.4: Monkeys (Primates), with taxonomic revisions of some forms. Proc. U. S. National Museum. 98:323-427.

22. IGAC. 1969. Monografía del departamento de Sucre. Oficina de estudios Geográficos. Instituto Agustín Codazzi (Bogotá, Colombia). 65p.

23. IZAWA, K. 1988. Preliminary reports on social changes on red howlers (Alouatta seniculus). Field Studies of New World Monkeys. La Macarena. 1:29-33.

24. JANSON, C.H.; GOLDSMITH, M.L. 1995. Predicting group size in primates: Foraging costs and predation risks. Behav. Ecol. 6:326-336.

25. JANSON, C.H. 1988. Intra-specific food competition and primate social structure: A synthesis. Behaviour. 105:117.

26. JANSON, C.H.; VAN SCHAIK, C. 1988. Recognizing the many faces of primate food competition: Methods. Behaviour. 105:165-186.

27. KATTAN, G.; ÁLVAREZ-LÓPEZ, H. 1996. Preservation and management of biodiversity in fragmented landscape in the Colombian Andes. En: Schelhas, J.; Greenberg, R. eds. Forest patches in tropical landscape. Island Press (Washington). p.3-18.
28. KLEIN, L.L.; KLEIN, D.J. 1976. Neotropical Primates: aspects of habitat usage, population density, and regional distribution in La Macarena, Colombia. En: Thorington, W.; Heltne, P.G. eds. Neotropical primates: Field studies and conservation. National Academy Press (Washington). p.70-78.

29. LAURANCE, W.; LOVEJOY, T.; VASCONCELOS, H.; BRUNA, E.; DIDHAM, R.; STOUFFER, P.; GASCON, C.; BIERREGAARD, R.; LAURANCE, S.; SAMPAIO, E. 2002. Ecosystem decay of Amazonian forest fragments: a 22-year investigation. Conserv. Biol. 16:605-618.

30. LEE, P.C. 1994. Social structure and evolution. En: Slater, P.B.; Halliday, T.R. eds. Behaviour and Evolution. Cambridge University Press (Cambridge). p.266-303.

31. MORALES-JIMÉNEZ, A.L. 2002. Densidad de los monos aulladores (Alouatta seniculus) en un bosque subandino, Risaralda, Colombia. Neotrop. Primates. 10(3):141-144.

32. NEVILLE, M.K. 1976. Alouatta seniculus. The population and conservation of howler monkeys in Venezuela. En: Thorington, R.W.; Heltne, P.G. eds. Neotropical Primates: Field studies and Conservation. Nal Acad. Sci. (Washington). p.101-109.

33. NEVILLE, M.K.; CASTRO, N.; MARMOLA, A.; REVILLA, J. 1976. Censusing primate population in the Reserve area of the Pacaya and Samiria Rivers, Department of Loreto, Perú. Primates. 17:151-181.

34. PERES, C.A. 1990. Effects of hunting on western Amazonian primate communities. Biol. Cons. 54:4759.

35. RONCANCIO, N.J.; GARCÍA, L.M.; ACOSTA, A. 2010. Densidad poblacional y estructura de grupo de Ateles hybridus brunneus (Primates: Atelidae) en un fragmento de bosque aislado en el suroriente de Antioquia, Colombia. Mastozoología Neotrop. 17(2):385-389.

36. SOINI, P. 1992. Ecología del coto mono (Alouatta seniculus, Cebidae) en el río Pacaya, Reserva nacional Pacaya-Samiria, Perú. Folia Amazónica. 4(2):103-118.

37. STERCK, E.H.M.; WATTS, D.P.; VAN SCHAIK, C.P. 1997. The evolution of female social relationships in nonhuman primates. Behav Ecol Sociobiol. 41:291309. 
38. STEVENSON, P. 1991. Relación entre la abundancia de frutos y las estrategias alimenticias de 4 especies de primates, en la Macarena - Colombia. Fundación para la Promoción de la Investigación y la Tecnología Banco de la República. Bogotá, Colombia. 209p.

39. STONER, K.E. 1994. Population Density of the Mantled Howler Monkey (Allouatta palliata) at La Selva Biological Reserve, Costa Rica: A new Technique to Analyze Census Data. Biotropica. 26(3):332-340.

40. TERBORGH, J.; JANSON, C.H. 1986. The socioecology of primate groups. Ann. Rev. Ecol. Syst. 17:111-136.

41. THORINGTON, R.W.; HELTNE, P.G. 1976. Introduction. En: Thorington, R.W.; Heltne, P.G. eds. Neotropical Primates: Field Studies and Conservation. Nal Acad. Sci. (Washington). p.1-3.

42. VALDERRAMA, C.; KATTAN, G. 2006. Plan de manejo del mono aullador rojo (Alouatta seniculus) en la región del Sirap. Eje Cafetero y valle del Cauca. Instituto de Investigación de Recursos Biológicos Alexander von Humboldt y Fundación EcoAndina/WCS Colombia (Bogotá). 115p.

43. VAN SCHAIK, C.P. 1989. The ecology of social relationships amongst female primates. En: Standen, V.; Foley, R.A. eds. Comparative Socioecology of Mammals and Humans (Oxford). p.195-218

44. WINTERHALDER, B.; SMITH, E.A. 1992. Evolutionary ecology and the social sciences. En: Smith, E.A.; Winterhalder, B. eds. Evolutionary Ecology and Human Behavior (New York). p.3-24.

45. ZAR, J.H. 1996. Bioestatistical Analysis. 3rd edition. Prentice-Hall, Inc. (Englewood Cliff, N.J). 663p.

Recibido: Agosto 3 de 2011

Aceptado: Octubre 9 de 2011 